\title{
Urgences
}

\section{Le corps évanoui. Sur René Crevel}

\section{Simon Harel}

Numéro 27, mars 1990

Images imaginaires

URI : https://id.erudit.org/iderudit/025576ar

DOI : https://doi.org/10.7202/025576ar

Aller au sommaire du numéro

Éditeur(s)

Urgences

ISSN

0226-9554 (imprimé)

1927-3924 (numérique)

Découvrir la revue

Citer cet article

Harel, S. (1990). Le corps évanoui. Sur René Crevel. Urgences, (27), 54-60.

https://doi.org/10.7202/025576ar d'utilisation que vous pouvez consulter en ligne.

https://apropos.erudit.org/fr/usagers/politique-dutilisation/ 


\section{Le corps évanoui. Sur René Crevel Simon Harel}

Ce qui en moi fut indéniable, je n'ai jamais eu la tentation d'en faire part à qui que ce soit. Au contraire l'instable, l'inquiet exigent une proclamation. La pensée en mouvement ne désire rien de plus que se figer dans une forme, car, de l'arrêt marqué, naît l'illusion de ce définitif dont la recherche est notre perpétuel tourment. 1

Cette remarque de Crevel, on la retrouve énoncée de façon lancinante tout au cours de l'élaboration de l'œuvre. Elle est d'autant plus dramatique que le suicide de Crevel s'apparente d'une certaine manière, avec toutes les nuances qui s'imposent, à la réalisation de cet "...arrêt marqué..." dans un "...perpétuel tourment... ». Propos qui circonscrivent l'impossible quête d'une écriture où l'affect occuperait une place centrale. Le plus beau témoignage étant certes Mon corps et moi, texte qui fait coïncider une relative mise à distance du sujet écrivant à l'égard de la constitution de son image du corps. C'est que la problématique du narcissisme est au cœur des écrits de Crevel. Ce dernier note à propos des peinture de Chirico:

Or si l'on peut dire que la tonalité de l'état d'âme courant résulte de la multitude des chocs affectifs, il faut noter que de tous les points qui figurent ces chocs, le plus "responsable " demeure le premier en date. 2

Puis :

Une solution ?... oui [à propos du suicide]. La mosaïque des simulacres ne tient pas. J'entends que l'ensemble des

1 René Crevel, Mon corps et moi (1926), Paris, Jean-Jacques Pauvert, 1979, p. 52.

2 René Crevel, "La minute qui s'arrête ou le bienfait de Giorgio de Chirico) " dans Mon corps et moi, dossier, op. cit, p. 193. 
combinaisons sociales ne saurait prévaloir contre l'angoisse dont est pétrie notre chair même. Aucun effort ne s'opposera jamais victorieusement à cette poussée profonde, à cet élan mystérieux, qui n'est point, $M$. Bergson, l'élan vital, mais son merveilleux contraire, l'élan mortel. 3

Une écriture de l'affect doit donc faire coïncider deux exigences distinctes. La recherche du premier choc affectif permettrait d'octroyer une régularité à cette multitude d'états d'âme imbriqués. Quête d'une *origine * qui est retour à un désir d'unité, constitution d'une mémoire du passé qui fait appel à la chronologie. Bien que Crevel mentionne dans un autre texte, à propos du suicide, cette «...mosaïque des simulacres... " sollicitée par l'élan mortel. Critique virulente à l'égard du bergsonisme pour lequel la référence psychologique à une conscience unifiante est déterminante. Chez Crevel, à chaque fois qu'une tentative de synthèse pourrait être évoquée, le désordre, le simulacre témoignent d'une écriture où la conscience de soi est tout au plus provisoire. Écriture où l'affect est le signe d'une tension, d'un affolement que le bergsonisme, critiquant les présupposés de l'Associationnisme, en vigueur à l'époque, ne peut circonscrire aisément 4 .

3 «Enquête. Le suicide est-il une solution. Réponse de René Crevel" (La révolution surréaliste, $\mathrm{n}^{\circ} 2,15$ janvier 1925) dans Mon corps et mol, dossier, op. cit., p. 199.

4 L'apport de Bergson est déterminant dès que l'on cherche à theoriser le statut de l'affectivité dans les discours littéraires de l'époque. Crevel y fait référence ironiquement en parlant d'élan mortel au lieu d'élan vital. Vocabulaire qui recoupe latéralement les recherches psychiatriques de l'entre-deux guerres. En témoignent notamment les travaux de Charles Blondel, de Guiraud, de Dide, de Targowla et de Dublineau.Selon Bercherie: "L'influence de Bergson est r ... indéniable sur l'ensemble de ces auteurs; il y a sans doute un certair alélisme entre les concepts de base de la phénoménologie allemarico et le bergsonisme (critique de l'Associationnisme, accent mis sur le rôle synthétique et fondateur de la conscience dans les phénomènes psychiques, attitude "compréhensive": description qualitative fine des états d'âme). "Paul Bercherie, Les fondements de la clinique. Histoire et structure du savoir psychiatrique, Paris, Navarin Editeur, Bibliothèque des Analytica, 1980, p. 270. Bercherie donne comme exemple de cette influence du bergsonisme la définition de la cénesthésie chez Blondel: «... sensibilité diffuse, ineffable qui sous-tend notre vécu et intègre notre affectivité el notre conscience corporelle. Habituellement réprimée par la conscience claire socio-discursive, elle produirait par son hypertrophie pathologique une modification profonde de la conscience, désormais irréductible aux valeurs collectives, devenue opaque aux catégories du langage, aux autres et à soi-même. "Bercherie, op.cit., p. 243. Car Bergson, toujours selon Bercherie, "... oppose les éléments que découpe dans la 
56

L'œuvre de Crevel, cette difficile narration de la sensation d'être, apparaît donc radicale. Elle fait référence, latéralement, aux hypothèses de Bergson pour mieux les contester. L'élément vital s'oppose à l'élan mortel, l'abstraction tempsespace, l'intuition durée-étendue, références constantes chez Bergson, ne trouvent pas de résonances réelles dans l'écriture de Crevel. Chez ce dernier, l'individualité de la conscience est un état tout au plus accidentel:

Au fur et à mesure que le jour m'éloigne du rêve nocturne, l'état qui en fut le résultat s'évaporant, je suis, pour le recréer, contraint de courir après un plus grand nombre d'images, de mots [...] Donc nous cherchons les sensations nettes et insuffisantes capables de recréer un état vague et suffisant. Je rêve d'un goût de chair humaine (non caressée ni mordue, mais mangée). Je me réveille avec une surprise dans la bouche. Comment y vint-elle ${ }^{5}$

Puis : "Ce qui revient à dire qu'un état premier se suffit à luimême...et ne demande secours ni à la philosophie ni à la littérature. Il se subit et n'a d'autre expression qu'un chant affectif interne et sans syllabes. " 6 Valorisation d'une dissociation du corps et de la psyché. Constat, ainsi que le note Crevel, d'une séparation entre "l'être" et "l'acte de pensée». Représentation d'une affectivité qui, chez Crevel, est toujours jouée in absentia. La perte, l'évanouissement, le suicide sont des états d'autant plus dangereux et séduisants qu'ils posent la question des limites de la conscience. Mais il y a néanmoins un destinataire constamment présent au cours de la création d'une œuvre dont le motif majeur est bien la surcharge affective. Le personnage maternel est perçu comme responsable de la mise à mort du père. Crevel le dit implicitement lorsqu'il associe l'émergence d'un désir matricide à cette "force majuscule», commodément identifiée à la nature ou à une présence divine, qui mena son père au suicide.

conscience, en une illusion de clarté, la pensée intelligente de caractère essentiellement pratique, spatial, qualitatif, social (avec son support essentiel: le langage) et la réalité psychologique qui lui est sous-jacente, essentiellement vécue dans la durée, diffuse, qualitative et individuelle. C'est l'occultation de la seconde par la première, la répression du "psychologique pur " qui permet l'intégration sociale, la réduction de l'individualité de la conscience au commun dénominateur du modèle spatial et du langage. " Paul Bercherie, op. cit., p. 243.

5 René Crevel, Mon corps et moj, op. cit., pp. 114-115.

6 René Crevel, op. cit., p. 53. 
Réponse à l'enquête de $L a$ révolution surréaliste qui est énoncée ainsi :

D'un suicide auquel il me fut donné d'assister, et dont l'auteuracteur était l'être, alors, le plus cher et le plus secourable à mon cœur, de ce suicide qui - pour ma formation ou ma déformation - fit plus que tout essai postérieur d'amour et de haine, dès la fin de mon enfance, j'ai senti que l'homme qui facilite sa mort est l'instrument docile et raisonnable d'une force majuscule (appelez-la Dieu ou Nature) qui, nous ayant mis au sein des médiocrités terrestres, emporte dans sa trajectoire, plus loin que ce globe d'attente, les seuls courageux. ${ }^{7}$

La présence paternelle est d'autant plus ambivalente chez Crevel qu'elle inaugure ce désir matricide. Les occurrences sont multiples, dans l'œuvre de Crevel, qui témoignent d'une représentation de la féminité sévère et violente, éprouvante et étouffante en l'absence d'un support symbolique paternel qui pourrait compenser, éloigner cette mère possessive qui provoque la fuite éperdue du fils. D'où la complainte de Crevel dans Mon corps et moi. La rage confessionnelle (la tentation autobiographique) est à la fois détestée et désirée. Dans les diverses autobiographies fictives de Crevel, ce n'est pas un Autre masculin qui est recherché obstinément. Pas simplement l'image commodément projetée sur l'Autre d'une fascination gémellaire, homosexuelle. Bien sûr cette perspective est présente dans l'œuvre de Crevel mais elle ne m'apparaît pas occuper tout l'espace que l'on voudrait à première vue lui attribuer. Crevel, dans son œuvre, fait plutôt l'expérience de l'absence du désir de l'Autre, constat tout à fait terrifiant.

Comme si l'œuvre créée était le signe d'une impasse: rencontre d'une mère incastrable, phallique qui étouffe son enfant, mère-contenant qui v(i)ole les pensées; amour homosexuel du fils piétinant ce corps maternel et qui simule ainsi, par déplacement, le désir de la mère pour un père absent. Car le désir des personnages masculins dans les écrits de Crevel mène souvent à l'échec. L'interrogation s'apparente à un soliloque angoissant. Qui suis-je? demande Crevel. Quel est ce corps

7 René Crevel, La révolution surréaliste, $n^{\circ} 2,15$ janvier 1925 dans Mon corps et moi, dossier, pp. 199-200. 
dont je m'épuise à revendiquer l'existence - grâce à la jouissance amoureuse - bien que je doute à chaque moment de sa (de ma) réalité. Le suicide apparaît alors, chez Crevel, comme une porte ouverte sur l'inconscient. N'emploie-t-il pas une expression de Breton significative. Faisant la recension du recueil de Breton Les pas perdus, il en extrait une phrase: «... pour moi se dérober si peu que ce soit à la règle psychologique équivaut à inventer de nouvelles façons de sentir... Je me suis toujours interdit de penser à l'avenir». Puis Crevel ajoute: «Se mettre à la fenêtre, c'est penser à l'avenir. Pour Breton se mettre à la fenêtre, c'est se jeter par la fenêtre. Le tout métaphoriquement d'ailleurs. " $8 \mathrm{La}$ dernière phrase de Crevel est moins innocente qu'il n'y paraît. Que signifie en effet un acte qui ne serait pas métaphorique. Sans doute un "état limite" qui correspondrait à une mise à l'épreuve: passage à l'acte que le suicide représentera. À cette condition, l'éprouvé d'affect pourra être saisi (ressenti) de manière à produire un acmé qui conjugue mort et vie. Le suicide définit cette alternative. Il est le doublet de la haine à l'égard de la «rage confessionnelle" dont Crevel fait état dans Le clavecin de Diderot. Alternance d'amour et de haine qui amène le sujet à se détruire pour s'être (re)connu trop vulnérable tel Narcisse. Se tuer, c'est aussi proférer un ultime vœu de mort à l'égard du destinataire maternel. Tue-moi dit Crevel (ou son porte-parole narratif) pour que j'éprouve dans son intensité maximale la haine que je te porte. À ce moment, oui, à ce moment seulement, il sera possible d'envisager une union mortifêre et incestueuse liant le fils à la mère, de même qu'un violent arrachement - une saisie définitive de la réalité dans sa contingence - qui disjoint le couple uni. C'est en effet dans la mort que le statut du destinataire maternel trouve sa pleine mesure. Pour Crevel un surplus de vie - de jouissance - est analogue à une mise à mort. Introjecter la mère phallique et dominatrice, omniprésente dans l'œuvre de Crevel, c'est du même coup effectuer un retour sur soi destructeur, affirmer le pouvoir d'une pulsion d'emprise mortifêre. Se tuer, c'est alors tuer la mère incastrable de l'inconscient à défaut de ne l'avoir pu aimer autrement que haineusement. L'intériorisation du vou matricide justifie le passage à l'acte. L'annihilation est la seule

8 René Crevel, "Les pas perdus par André Breton (N.R.F., editeur", Les feuilles libres, $n^{\circ} 36$, mars 1924, dans Mon corps et moi, dossier, op. cit., pp. 190-191. 
autre solution envisagée. L'intériorisation douloureuse du vœu matricide, l'identification ambivalente à la mère, la présence d'un père fantomatique suicidé - témoignage de l'inanité de la filiation paternelle - tels sont les différents motifs qui conduisent Crevel à rompre l'équilibre difficilement maintenu qui lui permettait d'écrire.

C'est que la reconnaissance du désir de l'Autre est reçue avec d'autant plus d'angoisse qu'elle implique le constat d'une séparation. Le drame d'un Crevel - et le caractère paroxystique de son œuvre - s'apparente alors à un incessant duel avec sa propre image sans que nul regard extérieur ne puisse authentifier qui est "soi" et qui est «autre». Vivre entre la mère haïe et le père absent, déchu, c'est se condamner à une perpétuelle fuite en avant que le suicide seul permettra de résoudre. Il n'est pas étonnant alors que l'œuvre de Crevel mette en scène avec tant d'insistance une interrogation sur le statut de l'identification spéculaire. C'est que le destinataire maternel est au cœur de l'œuvre, destinataire non pas aimé et désiré - en tant que manifestation du désir de l'Autre mais détesté parce qu'omniprésent. Mon corps et moi est le titre d'un des romans de Crevel. Comme s'il était possible de scinder l'affect de la pensée. Ambivalence de la distinction entre contenant et contenu. Qui suis-je, chez Crevel, signifie en fait "Quel corps puis-je habiter?"

Quête d'un narcissisme primaire, réponse immédiate à la demande de désir. Recherche douloureuse qui réactive une demande fusionnelle où le corps à corps avec la mère possède de forts accents mortiferes. Car chez Crevel la rencontre de la mère suppose toujours la persistance d'un meurtre reconduit: qui, de la mère ou du fils, devra payer pour le suicide du père? Le corps à corps est bien en effet une bataille à finir en l'absence du père qui pourrait s'interposer, se constituer à son tour comme objet de désir de la mère. L'intensité sensorielle, que l'on retrouve dans l'œuvre de Crevel, caractériserait donc une régression: quête d'un éprouvé d'affect dont l'écriture est appelée à subir les effets. Écriture semblable à un derme qui doit contenir et aussi figurer les traces d'un violent conflit avec la mère. D'où l'importance des motifs associés à la confession, à la symétrie. Fascination haineuse, miroitement narcissique qui peut être vécu en l'absence d'un père dépositaire des interdits. Qu'on pense à ces réflexions de Crevel sur la rage confessionnelle: 
60

... transpositions, transpositions, transpositions. - Et hardi petits! Nous aussi nous savons fabriquer de la fausse monnaie, des faux visages, des faux noms. Nous aussi nous allons écrire des romans, des confessions et servir une belle tranche de vie. Au travail. Demi-aveux, les pires mensonges. ${ }^{9}$

Refus de la confession, de la mise à nu qui accompagne avec ambivalence la constitution d'une autobiographie fictive. Crevel n'ajoute-il pas dans Êtes-vous fous? :

Je sais à quol m'en tenir et que je suis affligé non du classique complexe d'CEdipe, mais du simplexe anti-CEdipe... Je n'ai jamais voulu désirer ma mère... Or, malheur à l'homme qui n'a pas voulu coucher avec sa mère. 10

Refus de l'inceste...crainte haineuse du corps à corps avec la mère. Crainte d'autant plus forte qu'elle matérialise ce sentiment d'étouffement, de confusion narcissique désespérante que l'on retrouve dans son œuvre. Ne pas vouloir coucher avec sa mère parce que l'on fut violenté psychiquement par celle-ci, parce que l'on éprouva trop tôt, et de façon trop intense, la réalité d'un inceste langagier où les mots n'étaient pas partagés avec le père. Chez Crevel, le corps fait l'objet d'une nomination incessante. Interrogation lancinante sur les modalités de ce corps à corps. Peau maternelle, peau de l'enfant, peur du créateur, peau de l'œuvre à créer: l'affect est alors témoignage d'un arrachement, d'une sortie hors de la matrice maternelle, et d'un impossible détachement.

9 René Crevel, Mon corps et moi, op. cit., p. 58.

10 René Crevel, Êtes-vous fou? (1929), Paris, Gallimard, coll. "L'imaginaire ", 1984 , p. 118. 